\title{
Hahn-Banach extension theorem for interval-valued functions and existence of quasilinear functionals
}

\author{
Halise Levent and Yilmaz Yilmaz. \\ Department of Mathematics, Inonu University, 44280, Malatya, Turkey
}

Received: 28 September 2017, Accepted: 28 February 2018

Published online: 21 March 2018.

\begin{abstract}
In this paper we focus on developing bounded quasilinear interval-valued functions. We deal with the Hahn-Banach extension theorem for interval-valued functions. Finally, we show that there are enough bounded quasilinear functionals on the space of interval-valued functions to distinguish between its the regular elements.
\end{abstract}

Keywords: Quasilinear spaces, quasilinear operators, interval-valued functions, Hahn-Banach theorem.

\section{Introduction}

Interval-valued functions have a wide range of applications in several areas, e.g., chemical and structural engineering, economics, control circuity design, global optimization and signal processing. Interval analysis developed by Moore [1] plays an important role in applied mathematics and functional analysis. Interval-valued functions are discussed in several research papers,see for instance [2], [3], [4].

A closed interval denoted by $[a, b]$ is the set of real numbers given by $[a, b]=\{x \in \mathbb{R}: a \leq x \leq b\}$. If $a$ equals to $b$, namely, the interval contains a single real number then we say that $[a, b]$ is degenerate interval and it is shown as $\{a\}$ or $\{b\}$. The set of all nonempty intervals of real numbers is emblematized by $\Omega_{C}(\mathbb{R})$ and each element of $\Omega_{C}(\mathbb{R})$ is called a interval. Further, the intervals are excellent tools for handling global optimization problems. Further, we refer to [7], [6] and [1] for the global optimization theory and for some applications of interval analysis.

The Hahn-Banach extension theorem is one of the most important and fundamental theorems in the whole theory of normed spaces (see, [5]). The expression of such theorem is as follows.

Theorem 1. Let $X$ be a normed space, $Z$ a linear subspace of $X$ and $f$ a bounded linear functional on $Z$. Then there exists a bounded linear functional $\hat{f}$ on $X$ such that $f(x)=\hat{f}(x)$ for $x \in Z$ and $\|f\|=\|\hat{f}\|$.

In this paper we extend the Hahn-Banach theorem to interval-valued functions. Further, we show that the dual space of a consolidate normed quasilinear space (to illustrate the space of intervals) is richly supplied with bounded quasilinear functionals. For this it must be established an algebraic structure on the classes of interval-valued functions So it is was that, Aseev in 1986 present an approach for the function spaces of set-valued functions by raising the notion of "quasilinear space".

Let us give the definition of a quasilinear space which is presented by Aseev [8].

\footnotetext{
*Corresponding author e-mail: halisebanazili44@ gmail.com
} 
A set $X$ is called a quasilinear space (briefly; $Q L S$ ) if a partial order relation " $\preceq "$, an algebraic sum operation, and an operation of multiplication by real numbers are defined in it in such a way that the following conditions hold for any elements $x, y, z, v \in X$ and any $\alpha, \beta \in \mathbb{R}$,

$$
\begin{aligned}
x & \preceq x, \\
x & \preceq z \text { if } x \preceq y \text { and } y \preceq z, \\
x & =y \text { if } x \preceq y \text { and } y \preceq x, \\
x+y & =y+x, \\
x+(y+z) & =(x+y)+z,
\end{aligned}
$$

there exists an element (zero) $\theta \in X$ such that $x+\theta=x$,

$$
\begin{aligned}
\alpha(\beta x) & =(\alpha \beta) x, \\
\alpha(x+y) & =\alpha x+\alpha y, \\
1 x & =x, \\
0 x & =\theta, \\
(\alpha+\beta) x & \preceq \alpha x+\beta x, \\
x+z & \preceq y+v \text { if } x \preceq y \text { and } z \preceq v, \\
\alpha x & \preceq \alpha y \text { if } x \preceq y .
\end{aligned}
$$

Note that quasilinear spaces has been only introduced on the field $\mathbb{R}$, so far.

Any linear space is a QLS with the partial order relation defined by " $x \preceq y$ if and only if $x=y$ ". Perhaps the most popular example of a nonlinear QLS is $\Omega_{C}(\mathbb{R})$ with the inclusion relation " $\subseteq$ ", the algebraic sum operation

$$
x+y=[\underline{x}, \bar{x}]+[\underline{y}, \bar{y}]=[\underline{x}+\underline{y}, \bar{x}+\bar{y}]=\{a+b: a \in x, b \in y\}
$$

and the real-scalar multiplication

$$
\begin{aligned}
\lambda x & =\lambda[\underline{x}, \bar{x}]=\left\{\begin{array}{l}
{[\lambda \underline{x}, \lambda \bar{x}] \lambda \geqslant 0} \\
{[\lambda \bar{x}, \lambda \underline{x}] \lambda<0}
\end{array}\right. \\
& =\{\lambda a: a \in x\} .
\end{aligned}
$$

In fact $\Omega_{C}(\mathbb{R})$ is the set of all nonempty compact convex subsets of real numbers and it is a subset of $\Omega(\mathbb{R})$, the set of all nonempty compact subsets of real numbers which is another important example of a nonlinear QLS.

\section{Terminology and basic notations}

We will start this section by giving some basic results from [8].

Definition 1. An element $x^{\prime}$ is called inverse of $x \in X$ if $x+x^{\prime}=\theta$. The inverse is unique whenever it exists. An element $x$ possessing inverse is called regular, otherwise is called singular.

It will be assumed in what follows that $-x=(-1) x$. Note that $x^{\prime}$ may not be exists but if it exists then $x^{\prime}=-x$. An example of a singular element in the nonlinear QLS $\Omega_{C}(\mathbb{R})$ is the interval $[2,3]$ since $[2,3]+(-1)[2,3]=[-1,1] \neq \theta$. However $-[2,3]=[-3,-2] \in \Omega_{C}(\mathbb{R})$.Further, a characterization of a regular element. $x$ is that $x^{\prime}=-x$, or equivalently, $x-x=\theta$. For example, the degenerate interval $\{3\}$ is a regular element in $\Omega_{C}(\mathbb{R})$. We should note that in a linear QLS, 
briefly in a linear space, each element is regular. Hence the notions of regular and singular elements in linear spaces are redundant.

Lemma 1. [8] Suppose that each element $x$ in $Q L S X$ has inverse element $x^{\prime} \in X$. Then the partial order in $X$ is determined by equality, the distributivity conditions hold, and consequently $X$ is a linear space.

In a real linear space, the equality is the only way to define a partial order such that conditions (1)-(13) hold.

Definition 2. [10] Suppose that $X$ is a $Q L S$ and $Y \subseteq X$. Then $Y$ is called a subspace of $X$ whenever $Y$ is a $Q L S$ with the same partial order and the restriction to $Y$ of the operations on $X$.

It is shown in [10].that $\Omega_{C}(\mathbb{R})$ is a subspace of $\Omega(\mathbb{R})$.

Let $Y$ be a subspace of a QLS $X$ and suppose each element $x$ in $Y$ has an inverse in $Y$. Then by Lemma 1 the partial order on $Y$ is determined by the equality. In this case $Y$ is a linear subspace of $X$.

An element $x$ in $X$ is said to be symmetric if $-x=x$ and $X_{\text {sym }}$ denotes the set of all symmetric elements. In a linear QLS, equivalently, in a linear space zero is the only symmetric element. $X_{r}$ and $X_{S}$ stand for the set of all regular, and singular elements with zero in $X$, respectively. Further, it can be easily shown that $X_{r}, X_{s y m}$ and $X_{s}$ are subspaces of $X$. They are called regular, symmetric and singular subspaces of $X$, respectively. Furthermore, it isn't hard to prove that summation of a regular element with a singular element is a singular element. Regular subspace of $X$ is a linear space while the singular subspace is a nonlinear QLS.

Example 1. In $\Omega_{C}(\mathbb{R})$,

$$
X_{s}=\{0\} \cup\{[a, b]: a, b \in \mathbb{R} \text { and } a<b\}
$$

is the singular subspace of $X$. Further $\{[a, b]=\{a\}: a \in \mathbb{R}\}$ is the set of all degenerate intervals or of the set of all singletons of $\mathbb{R}$ constitutes the regular subspace $X_{r}$. It is a linear subspace of $\Omega_{C}(\mathbb{R})$ and $X_{r}$ is the copy of $\mathbb{R}$ in $\Omega_{C}(\mathbb{R})$. Symmetric subspace

$$
X_{\text {sym }}=\{[-a, a]: a \in \mathbb{R}\}
$$

of $\Omega_{C}(\mathbb{R})$ is also a subspace of $X_{s}$. In a linear space $X, X_{s y m}=X_{s}=\{\theta\}$, the trivial subspace, and $X_{r}=X$.

In a QLS $X$, a real function $\|\cdot\|_{X}: X \longrightarrow \mathbb{R}$ is called a norm if the following conditions hold [8]:

$$
\begin{gathered}
\|x\|_{X}>0 \text { if } x \neq 0, \\
\|x+y\|_{X} \leq\|x\|_{X}+\|y\|_{X}, \\
\|\alpha x\|_{X}=|\alpha|\|x\|_{X}, \\
\text { if } x \preceq y, \text { then }\|x\|_{X} \leq\|y\|_{X},
\end{gathered}
$$

if for any $\varepsilon>0$ there exists an element $x_{\varepsilon} \in X$ such that

$$
x \preceq y+x_{\varepsilon} \text { and }\left\|x_{\mathcal{E}}\right\|_{X} \leq \varepsilon \text { then } x \preceq y .
$$

A quasilinear space $X$ with a norm defined on it, is called normed quasilinear space (briefly, normed $Q L S$ ). It follows from Lemma 2 that if any $x \in X$ has an inverse element $x^{\prime} \in X$ then the concept of normed QLS coincides with the concept 
of real normed linear space. Hausdorff metric or norm metric on $X$ is defined by the equality

$$
h_{X}(x, y)=\inf \left\{r \geq 0: x \preceq y+a_{1}^{r}, y \preceq x+a_{2}^{r} \text { and }\left\|a_{i}^{r}\right\| \leq r, i=1,2\right\}
$$

Since $x \preceq y+(x-y)$ and $y \preceq x+(y-x)$, the quantity $h_{X}(x, y)$ is well-defined for any elements $x, y \in X$, and the function $h_{X}$ satisfies all axioms of the metric. Further $h_{X}(x, y)$ may not equal to $\|x-y\|_{X}$ if $X$ is not a linear space but always $h_{X}(x, y) \leq\|x-y\|_{X}$ for every $x, y \in X[8]$.

Example 2. [8] For a normed linear space $E$, a norm on $\Omega(E)$ is defined by

$$
\|A\|_{\Omega}=\sup _{a \in E}\|a\|_{E}
$$

Hence $\Omega_{C}(E)$ and $\Omega(E)$ are normed quasilinear spaces. In this case the Hausdorff (norm) metric is defined as usual:

$$
h(x, y)=\inf \left\{r \geq 0: x \subseteq y+S_{r}(\theta), y \subseteq x+S_{r}(\theta)\right\}
$$

where $S_{r}(\theta)$ is closed ball of $E$ and $x, y$ are elements of $\Omega_{C}(E)$ or $\Omega(E)$.

Partial ordering structure of the QLS plays again a crucial role in this section. QLSs may have various types. We want to describe best advantages one.

Definition 3. [9] Let $X$ be a quasilinear space, $M \subseteq X$ and $x \in M$. The set

$$
F_{x}^{M}=\left\{z \in M_{r}: z \preceq x\right\}
$$

is called floor in $M$ of $x$. In the case of $M=X$ it is called only floor of $x$ and written briefly $F_{x}$ instead of $F_{x}^{X}$.

Floor of an element $x$ in linear spaces is the singleton $\{x\}$. Therefore, it is nothing to discuss the notion of floor of an element in a linear space.

Definition 4. [9] Let $X$ be a quasilinear space and $M \subseteq X$. Then the union set

$$
\bigcup_{x \in M} F_{x}^{M}
$$

is called floor of $M$ and is denoted by $F_{M}$. In the case of $M=X, F_{X}$ is called floor of the quasilinear space $X$.

Definition 5. [9] A quasilinear space $X$ is called consolidate quasilinear space whenever sup $F_{y}$ exists for every $y \in X$ and

$$
y=\sup F_{y}=\sup \left\{z \in X_{r}: z \preceq y\right\} .
$$

Otherwise, $X$ is called non-consolidate quasilinear space.

Especially, we should note that the supremum in this definition is defined according to the partial order relation “ $\preceq$ ” on $X$.

For any normed linear space $E, \Omega(E)$ and $\Omega_{C}(E)$ are consolidate normed quasilinear space. Further, singular subspace

$$
\mathscr{A}=\{0\} \cup\{[a, b]: a \neq b, a, b \in \mathbb{R}\}
$$

of $\Omega_{C}(\mathbb{R})$ is a nc-QLS since $F_{y}=\emptyset$ for $y=[1,2]$ in $A$. Further,

$$
\mathscr{B}=\{[a, b]: a \leq 0 \leq b, a, b, 0 \in \mathbb{R}\}
$$


is another nc-subspace of $\Omega_{C}(\mathbb{R})$.

Remark. In general, a nonlinear QLS $X$ consists of its regular part $X_{r}$ which is a linear space and its singular part $X_{s}$ which is an nc-QLS.

\section{Some new results on interval-valued functions}

Let us start this section by recording some concepts and results on quasilinear operators given by Aseev.

Definition 6. [8] Let $X$ and $Y$ be quasilinear spaces. A mapping $T: X \rightarrow Y$ is called a quasilinear operator if it satisfies the following conditions:

$$
\begin{gathered}
T\left(x_{1}+x_{2}\right) \preceq T\left(x_{1}\right)+T\left(x_{2}\right), \\
T(\alpha x)=\alpha T(x) \text { for any } \alpha \in \mathbb{R}, \\
\text { if } x_{1} \preceq x_{2} \text {, then } T\left(x_{1}\right) \preceq T\left(x_{2}\right) .
\end{gathered}
$$

If $X$ and $Y$ are linear spaces, by Lemma 1 we say that the definition of a quasilinear operator coincides with the usual definition of a linear operator. The last condition is automatically satisfied in this case.

Definition 7. [8] Let $X$ and $Y$ be a normed quasilinear spaces. A quasilinear operator $T: X \rightarrow Y$ is said to be bounded if there exists a number $k>0$ such that $\|T x\| \leq k\|x\|$ for any $x \in X$.

Lemma 2. [8] Suppose that $X$ and $Y$ are normed quasilinear spaces. Then a quasilinear operator $T: X \rightarrow Y$ is bounded if and only if it is continuous at the point $\theta \in X$. The continuity of $T$ at $\theta$ implies that it is uniformly continuous on $X$.

Let $X$ and $Y$ be a normed quasilinear spaces. Denote by $\Lambda(X, Y)$ the space of all bounded quasilinear operators from $X$ to $Y$. We write $T_{1} \preceq T_{2}$ if and only if $T_{1}(x) \preceq T_{2}(x)$ for any $x \in X$. Algebraic sum and multiplication by real numbers are defined on $\Lambda(X, Y)$ as follows; $\left(T_{1}+T_{2}\right)(x)=T_{1}(x)+T_{2}(x)$ and $(\alpha T)(x)=\alpha T(x)$, for $x \in X$. Therefore $\Lambda(X, Y)$ is a quasilinear space with these operations and partial order relation. The norm on $\Lambda(X, Y)$ is defined by $\|T\|_{\Lambda}=\sup _{\|x\|=1}\|T(x)\|_{Y}$. Hence $\Lambda(X, Y)$ is a normed quasilinear space [8].

Suppose that $X$ is a normed quasilinear space. Then the space $\Lambda(X, \Omega(\mathbb{R}))$ is called the dual space of $X$ and is denoted $X^{\star}$. Further, the space $\Lambda\left(X, \Omega_{C}(\mathbb{R})\right)$ is called the convex dual space of $X$ and is denoted $X_{C}^{\star}$. Any quasilinear operator from $X$ to $\Omega(\mathbb{R})$ is called a quasilinear functional [8]. Also, a quasilinear operator from $X$ to $\Omega_{C}(\mathbb{R})$ is said a convex quasilinear functional (briefly, c-quasilinear functional).

Note that a quasilinear functional is an operator whose range lies on the $\Omega(\mathbb{R})$, i.e., the range its is not $\mathbb{R}$. Because if $X$ is a space of subset (for example $\Omega_{C}(\mathbb{R})$ ), then $\Lambda(X, \mathbb{R})$ consists of the single element $\{\theta\}$.

Example 3. [8] (A example on interval-valued function) Let $X$ be a linear space and let the function $p: X \rightarrow \mathbb{R}$ be sublinear, i.e., the following conditions hold:

$$
\begin{gathered}
p(\alpha x)=\alpha p(x), \alpha \geq 0, \\
p\left(x_{1}+x_{2}\right) \leq p\left(x_{1}\right)+p\left(x_{2}\right) .
\end{gathered}
$$

Then the mapping $\varphi: X \rightarrow \Omega_{C}(\mathbb{R})$ defined by $\varphi(x)=[-p(-x), p(x)]$ is a quasilinear functional from $X$ to $\Omega_{C}(\mathbb{R})$. If $X$ is a normed linear space and $p$ is continuous, then $\varphi \in X_{C}^{\star}$. Conversely, suppose that $\varphi: X \rightarrow \Omega_{C}(\mathbb{R})$ is a quasilinear 
operator, where $X$ is a linear space. Then the interval $\left[f_{1}(x), f_{2}(x)\right]$ is the value of $\varphi(x)$ for any $x \in X$. It follows from the quasilinearity of the operator $\varphi$ that if $\alpha \geq 0$,

$$
\begin{gathered}
f_{2}(\alpha x)=\alpha f_{2}(x), \\
f_{2}\left(x_{1}+x_{2}\right) \leq f_{1}\left(x_{1}\right)+f_{2}\left(x_{2}\right)
\end{gathered}
$$

and

$$
f_{1}(x)=-f_{2}(-x)
$$

Consequently, there exists a unique sublinear function $p(x)=f_{2}(x)$ such that $\varphi(x)=[-p(-x), p(x)]$. If $X$ is a normed linear space and $\varphi \in X_{C}^{\star}$, then the corresponding sublinear function $p$ is continuous.

Theorem 2. Any element $T \in \mathbb{R}_{C}^{\star}$ can uniquely be represented by

$$
T(x)=x A
$$

where $A \in \Omega_{C}(\mathbb{R})$.

Proof. Suppose that $T \in \mathbb{R}_{C}^{\star}$. Every $x \in \mathbb{R}$ has an unique representation such that

$$
x=x 1
$$

where $\{1\}$ is the standard basis of $\mathbb{R}$. Further, $T(x)=T(1 x)=x T(1)$ since $T$ is a quasilinear operator. If we take $T(1)=$ $A \in \Omega_{C}(\mathbb{R})$ then

$$
T(x)=x A
$$

Conversely, let $T: \mathbb{R} \rightarrow \Omega_{C}(\mathbb{R})$ be defined by $T(x)=x A$ where the interval $A$ is a distinguished element of $\Omega_{C}(\mathbb{R})$. First of all, $T$ is well-defined. Indeed; $T x=x A=\{x a: a \in A\} \in \Omega_{C}(\mathbb{R})$ for $A \in \Omega_{C}(\mathbb{R})$. Furthermore, $T$ is a quasilinear operator: For any $x, y \in \mathbb{R}, \lambda \in \mathbb{R}$ and $A \in \Omega_{C}(\mathbb{R})$,

$$
\begin{gathered}
T(x+y)=(x+y) A \subseteq x A+y A=T(x)+T(y), \\
T(\lambda x)=(\lambda x) A=\lambda(x A)=\lambda T(x)
\end{gathered}
$$

and

$$
\text { If } x=y \text { then } T(x)=x A=y A=T(y) \text { and so } T(x) \subseteq T(y) \text {. }
$$

It is obvious that (19) representation is unique.

Theorem 3. The space $\mathbb{R}_{C}^{\star}$ is a consolidate QLS.

Proof. By the Theorem 2, we know that any elements $f \in \mathbb{R}_{C}^{\star}$ has the representation

$$
f(x)=x[u, v]
$$

for an element $[u, v] \in \Omega_{C}(\mathbb{R})$ and this representation is unique. Firstly, we determine the regular elements of preceding from $f$. Let us describe the function $g_{a}: \mathbb{R} \rightarrow \Omega_{C}(\mathbb{R})$ by the equality $g_{a}(x)=a .\{x\}$ for a constant element $a \in[u, v]$ and $x \in \mathbb{R}$. It is not hard to see that $g \in \Lambda\left(\mathbb{R}, \Omega_{C}(\mathbb{R})\right)_{r}$. Further, for every $x \in \mathbb{R}, a .\{x\} \subseteq x .[u, v]$ and so for each element $a \in[u, v]$ we have that $g_{a} \leq f$. We must show $\sup \left\{g_{a}: a \in[u, v]\right\}=f$ to complete the proof. It is obvious that the set $\left\{g_{a}: a \in[u, v]\right\}$ is bounded and $f$ is an upper bound for this set. Suppose that the element $h$ is another upper bounded of the set $\left\{g_{a}: a \in[u, v]\right\}$. Let us assume that $f \not \leq h$. There exists an element $x_{0} \in \mathbb{R}$ such that $f\left(x_{0}\right) \nsubseteq h\left(x_{0}\right)$. This means that $t_{0} \in f\left(x_{0}\right)$ but $t_{0} \notin h\left(x_{0}\right)$ for $t_{0} \in \mathbb{R}$. Since $t_{0} \in f\left(x_{0}\right)$, there exists an element $a \in[u, v]$ such that $t_{0}=x_{0} a$. If $a=0$ 
then $g_{a}(x)=g_{0}(x)=0\{x\}=\{0\}$ for every $x \in \mathbb{R}$ and especially $g_{a}\left(x_{0}\right)=\{0\}$ for $x=x_{0}$. If $a=0$ then $t_{0}=0$ and $\left\{t_{0}\right\}=\{0\} \nsubseteq h\left(x_{0}\right)$ and so $g_{a}\left(x_{0}\right) \nsubseteq h\left(x_{0}\right)$ since $t_{0}=0 \notin h\left(x_{0}\right)$. Therefore, we obtain $g_{a} \not \leq h$. If $a \neq 0$ then we write $x_{0}=\frac{t_{0}}{a}$ by the equality $t_{0}=x_{0} a$. Further,

$$
g_{a}\left(x_{0}\right)=a\left\{x_{0}\right\}=a\left\{\frac{t_{0}}{a}\right\}=\left\{t_{0}\right\}
$$

and so $g_{a}\left(x_{0}\right) \nsubseteq h\left(x_{0}\right)$, since $\left\{t_{0}\right\} \notin h\left(x_{0}\right)$. Thus we say that $g_{a} \not h$. Eventually this contradicts with the fact that $f$ is an upper bound of $\left\{g_{a}: a \in[u, v]\right\}$. Since $h$ is an arbitrary element, we have that $\sup \left\{g_{a}: a \in[u, v]\right\}=f$.

\section{The Hahn-Banach theorem}

Theorem 4. Let $X$ be a linear space and $\varphi$ be a c-quasilinear functional defined on $X$. Suppose that $Z$ is a subspace of $X$ and $f$ is a c-quasilinear functional defined on $Z$ such that

$$
\|f(x)\|_{\Omega} \leq\|\varphi(x)\|_{\Omega}
$$

for all $x \in Z$. Then, there exists a quasilinear extension $\tilde{f}: X \rightarrow \Omega_{C}(\mathbb{R})$ of $f($ i.e. $f(x)=\tilde{f}(x)$ for all $x \in Z$ ) such that

$$
\|\tilde{f}(x)\|_{\Omega} \leq\|\varphi(x)\|_{\Omega}
$$

for all $x \in X$.

Proof. The proof involves three steps:

Step 1. Let $P$ be the set of all quasilinear extensions $g: Y \rightarrow \Omega_{C}(\mathbb{R})$ of $f$ satisfied the condition

$$
\|g(x)\|_{\Omega} \leq\|\varphi(x)\|_{\Omega}
$$

for all $x \in Y$, where $Y$ is subspace of $X$. Clearly $P$ is non-empty since $f \in P$. Consider the partial order relation defined on $P$ by

$$
g \lesssim \tilde{g}
$$

if $Y \subset \tilde{Y}$ and $\tilde{g}: \tilde{Y} \rightarrow \Omega_{C}(\mathbb{R})$ is a quasilinear extension of $g$. Let $C$ be a chain in $P$. Define

$$
Y=\cup_{i \in I} Y_{i}
$$

where $Y_{i}$ is subspace of $X$. Let $g: Y \rightarrow \Omega_{C}(\mathbb{R})$ be defined by $g(x)=g_{i}(x)$ if $x \in Y_{i}$, where $g_{i}: Y_{i} \rightarrow \Omega_{C}(\mathbb{R})$ is an element of $C$ for each $i=1,2, \ldots$. Since $C$ is a chain, it is not hard to see that $g$ is well defined and also that is a quasilinear extension of each of $g_{i}$. Also $\|g(x)\|_{\Omega} \leq\|\varphi(x)\|_{\Omega}$ for all $x \in Y$. Hence $g$ is an upper bound of $C$. Since $C \subset P$ is arbitrary, $P$ has a maximal element $\tilde{f}$ by Zorn's lemma. By the definition of $P$, this is a quasilinear extension of $f$ which satisfies

$$
\|\tilde{f}(x)\|_{\Omega} \leq\|\varphi(x)\|_{\Omega}
$$

for all $x \in D(\tilde{f}) .(D(\tilde{f})$ is the domain set of $\tilde{f}$.)

Step 2. We will show that $D(\tilde{f})=X$. Suppose that $D(\tilde{f}) \neq X$. Then we can choose a $y_{1} \in X \backslash D(\tilde{f})$. Note that $y_{1} \neq 0$ since $0 \in D(\tilde{f})$. Consider the subspace $Y_{1}$ of $X$ given by

$$
Y_{1}=\left\{y+\alpha y_{1}: y \in D(\tilde{f}), \alpha \in \mathbb{R}\right\}
$$


Now we will define a function $g_{1}: Y_{1} \rightarrow \Omega_{C}(\mathbb{R})$ such that

$$
g_{1}\left(y+\alpha y_{1}\right)=\left\{\begin{array}{cc}
\tilde{f}(y) & \alpha=0 \\
\varphi\left(y+\alpha y_{1}\right) & \alpha \neq 0
\end{array}\right.
$$

Let us remember that any $x \in Y_{1}$ can be written $x=y+\alpha y_{1}$ for $y \in D(\tilde{f})$. Since this representation is unique, $g_{1}$ is well defined. Also it is immediate to see that $g_{1}$ is quasilinear, since the functions $\tilde{f}$ and $\varphi$ are quasilinear. Furthermore, for $\alpha=0$ we have $g_{1}(y)=\tilde{f}(y)$. Hence $g_{1}$ is an extension of $\tilde{f}$ such that $D(\tilde{f})$ is a proper subset of $Y_{1}$. Consequently, if we can prove that $g_{1} \in P$ by showing that

$$
\left\|g_{1}(x)\right\|_{\Omega} \leq\|\varphi(x)\|_{\Omega}
$$

for all $x \in Y_{1}$ this will contradict the maximality of $\tilde{f}$. Thus we will prove that $D(\tilde{f})=X$.

Step 3. For all $x \in Y_{1}$, if $\alpha=0$ then

$$
\left\|g_{1}(x)\right\|_{\Omega}=\left\|g_{1}\left(y+\alpha y_{1}\right)\right\|_{\Omega}=\|\tilde{f}(y)\|_{\Omega} .
$$

Since $\tilde{f}$ is a quasilinear extension of $f$ which satisfies $\|\tilde{f}(x)\|_{\Omega} \leq\|\varphi(x)\|_{\Omega}$ for all $x \in D(\tilde{f})$, we have that $\|\tilde{f}(y)\|_{\Omega} \leq$ $\|\varphi(y)\|_{\Omega}$ for all $y=x \in D(\tilde{f}) \subset Y_{1}$ and so $\left\|g_{1}(x)\right\|_{\Omega} \leq\|\varphi(x)\|_{\Omega}$. Similarly, by considering $\alpha \neq 0$, we write that

$$
\left\|g_{1}(x)\right\|_{\Omega}=\left\|g_{1}\left(y+\alpha y_{1}\right)\right\|_{\Omega}=\left\|\varphi\left(y+\alpha y_{1}\right)\right\|_{\Omega}=\|\varphi(x)\|_{\Omega}
$$

and also that $\left\|g_{1}(x)\right\|_{\Omega} \leq\|\varphi(x)\|_{\Omega}$. Hence the proof is complete.

Theorem 5. Let $Z$ be a subspace of real normed linear space $X$ and assume that $f$ is a bounded c-quasilinear functional on $Z$. Then there exists a bounded quasilinear extension $\tilde{f}: X \rightarrow \Omega_{C}(\mathbb{R})$ of $f$ such that

$$
\|f\|_{\Lambda}=\|\tilde{f}\|_{\Lambda}
$$

where $\|\tilde{f}\|_{\Lambda}=\sup _{x \in X,\|x\|=1}\|\tilde{f}(x)\|_{\Omega}$ and $\|f\|_{\Lambda}=\sup _{x \in Z,\|x\|=1}\|\tilde{f}(x)\|_{\Omega}$

Proof. If $Z=\{0\}$, then $f=0$, and so $\tilde{f}=0$. Let $Z \neq\{0\}$. We know that

$$
\|f(x)\|_{\Omega} \leq\|f\|_{\Lambda}\|x\|
$$

for all $x \in Z$. Define $p: X \rightarrow \mathbb{R}$ such that $p(x)=\|f\|\|x\|$ for all $x \in X . p$ is a sublinear functional on $X$, since

$$
p(x+y)=\|f\|\|x+y\| \leq\|f\|(\|x\|+\|y\|)=p(x)+p(y)
$$

and

$$
p(\alpha x)=\|f\|\|\alpha x\|=|\alpha|\|f\|\|x\|=|\alpha| p(x) .
$$

On the other hand, by the Example 3, the mapping $p: X \rightarrow \mathbb{R}$ defines a mapping $\varphi: X \rightarrow \Omega_{C}(\mathbb{R})$ by $\varphi(x)=[-p(-x), p(x)]$ such that $\varphi$ is a quasilinear operator. Furthermore, we can write $\|f(x)\|_{\Omega} \leq|p(x)|$ by the definition of $p$. Since

$$
\|\varphi(x)\|_{\Omega}=\|[-p(-x), p(x)]\|_{\Omega}=\|[-p(x), p(x)]\|_{\Omega}=|p(x)|,
$$


we say that $\|f(x)\|_{\Omega} \leq\|\varphi(x)\|_{\Omega}$. Hence we can now apply Theorem 4 and conclude that there exists a quasilinear functional $\tilde{f}$ on $X$ which is an extension of $f$ and satisfies

$$
\|\tilde{f}(x)\|_{\Omega} \leq\|\varphi(x)\|_{\Omega}=\|f\|\|x\|
$$

for all $x \in X$. Taking the supremum over all $x \in X$ of norm 1 , we obtain the inequality

$$
\|\tilde{f}\|_{\Lambda}=\sup _{x \in X,\|x\|=1}\|\tilde{f}(x)\|_{\Omega} \leq\|\tilde{f}\|_{\Lambda}=\sup _{x \in X,\|x\|=1}\|f\|\|x\|=\|f\| .
$$

Since under an extension the norm cannot decrease, we also have $\|f\|_{\Lambda} \leq\|\tilde{f}\|_{\Lambda}$ and so the theorem is proved.

The Hahn-Banach theorem implies that the convex dual space $X_{C}^{\star}$ of a normed quasilinear space $X$ consists of many bounded c-quasilinear functionals to distinguish between the regular elements of $X$.

Theorem 6. Let $X$ be a consolidate normed quasilinear space and $x_{0} \in X$ a non-zero regular element. Then there exists a bounded c-quasilinear functional $\tilde{f}$ on $X$ satisfying $\|\tilde{f}\|=1$ and $\tilde{f}\left(x_{0}\right)=\left\|x_{0}\right\|$.

Proof. Let $Z$ be the one-dimensional space spanned by $x_{0}$, i.e., $Z$ consists of all elements $x=\alpha x_{0}$ where $\alpha$ is a real scalar. It is obvious that $Z$ is a subspace of $X_{r}$. Define a c-quasilinear functional $f$ on $Z$ by

$$
f(x)=f\left(\alpha x_{0}\right)=\left\{\alpha\left\|x_{0}\right\|\right\}
$$

Since

$$
\|f(x)\|_{\Omega}=\left\|f\left(\alpha x_{0}\right)\right\|=\left\|\left\{\alpha\left\|x_{0}\right\|\right\}\right\|_{\Omega}=|\alpha|\left\|x_{0}\right\|=\left\|\alpha x_{0}\right\|=\|x\|,
$$

$f$ is bounded and has norm $\|f\|=1$. Theorem 5 implies that $f$ has a quasilinear extension $\tilde{f}$ from $Z$ to $X_{r}$ with norm $\|f\|_{\Lambda}=\|\tilde{f}\|_{\Lambda}=1$. From (20), we say that

$$
\tilde{f}\left(x_{0}\right)=f\left(x_{0}\right)=\left\|x_{0}\right\| .
$$

Corollary 1. Let $X$ be a consolidate normed quasilinear space. If $X \neq\{0\}$ then $X_{C}^{\star}$ cannot be $\{0\}$. Of course, $X^{\star}$ also cannot be $\{0\}$ since $X_{C}^{\star} \subset X^{\star}$.

\section{Competing interests}

The authors declare that they have no competing interests.

\section{Authors' contributions}

All authors have contributed to all parts of the article. All authors read and approved the final manuscript.

\section{References}

[1] R. E. Moore, R. B. Kearfott, M. J. Cloud, Introduction to Interval Analysis, SIAM, Philadelphia, 2009.

[2] G. Alefeld, G. Mayer, Interval Analysis: theory and applications, Journal of Computational and Applied Mathematics, 121 (2000), 421-464.

[3] A. Neumaier, Interval Methods for Systems of Equations, Cambridge Universty Press (1990).

[4] R. B. Kearfott, V. Kreinovich (Eds.), Applications of interval computations, Kluwer Academic Publishers, 1996. 
[5] E. Kreyszing, Introductory functional analysis with applications, Canada, 1978.

[6] H. Ratschek, J. Rokne, New computer methods for global optimization, Ellis Horwood Series: Mathematics and its Applications. Ellis Horwood Ltd., Chichester; Halsted Press [JohnWiley \&Sons, Inc.], New York, 1988.

[7] V. Lakshmikantham, T. Gana Bhaskar, J. Vasandura Devi, Theory of set differential equations in metric spaces, Cambridge Scientific Publishers, Cambridge, 2006.

[8] S.M. Aseev, Quasilinear operators and their application in the theory of multivalued mappings, Proceedings of the Steklov Institute of Mathematics, Issue 2, 23-52, 1986.

[9] H. Bozkurt, Y. Y1lmaz, Some New Results on Inner Product Quasilinear Spaces, Cogents Mathematics, 3 (2016),1194801.

[10] Y. Yılmaz, S. Çakan, Ş. Aytekin, Topological Quasilinear Spaces, Abstr. Appl. Anal., (2012), Article ID 951374, 10 pages. 